\title{
MAKER: Designing and Building a Prosthetic Hand for a High School Engi- neering Design Course
}

\section{Mr. Seref Yagli, Harmony Public School}

I have coached four different robotics clubs this school year, which are: Wex's robotics club, FTC (First Tech Challenge) club, FRC (First Robotics Competition), and SeaPerch Underwater Robotics. My teams have won championships, as well as technical and designing awards at FLL competitions. I also began teaching Robotics and Automation and Concepts of Engineering and Technology, classes in which students learn to apply engineering and designing skills as well as robotic coding. I am a former member of Dallas Robotics Group at the Dallas Makerspace, and plan to enroll in the National Stem certification program. I have completed PLTW and Robotics and Automation certificate programs, and attended Arduino Project and Raspberry Pie. I have been a mentor for the College Readiness and Leadership Program (CRLP) with the goal of implementing student leadership in our school. In addition, I like to work with 3D printing and design, and am a member of Enabling Hand, a team that creates and assembles prosthetic arm designs.

\section{Dr. Sheng-Jen "Tony" Hsieh, Texas A\&M University}

Dr. Sheng-Jen ("Tony") Hsieh is a Professor in the Dwight Look College of Engineering at Texas A\&M University. He holds a joint appointment with the Department of Engineering Technology and the Department of Mechanical Engineering. His research interests include engineering education, cognitive task analysis, automation, robotics and control, intelligent manufacturing system design, and micro/nano manufacturing. He is also the Director of the Rockwell Automation laboratory at Texas A\&M University, a state-of-the-art facility for education and research in the areas of automation, control, and automated system integration. 


\title{
MAKER: Designing and Building a Prosthetic Hand for a High School Engineering Design Course
}

\begin{abstract}
This paper describes a high school-level engineering design project in which students design and build a prosthetic hand. Students use Tinkercad to modify a basic 3D hand design, print the parts using a 3D printer, and assemble the hand. They then integrate the hand with electromyography (EMG) sensors and an Arduino board. The EMG sensors detect arm muscle activation via electric potential. The Arduino board translates the EMG signals into finger movements. The final goal is successful execution of a daily-life task such as picking up a piece of paper, paper money, or a coin; holding a water bottle or a pen/pencil/paint brush; or gripping a toothbrush. The project allows students to learn about CAD, 3D printing, sensors, and Arduino programming in the context of solving a real-world problem, and helps raise their awareness of disabilities. The equipment cost for the project is less than $\$ 150$ per hand assembly.
\end{abstract}

\section{Introduction}

Prosthetic limb design has greatly improved over the last decade however; it still remains inaccessible to general population. The purpose of this project to design and build affordable functional prosthetic hand controlled by muscle movement in person's arm and helping children from low-income families who have a disabled hand and creating social consciousness on students.

The module will be used in Engineering Tech classes and as an extracurricular activity. The modules consist of the prosthetic arm design, 3D printing and assembling the hand, and programming the Arduino microcontroller to control the prosthetic limb according muscle movements in the arm [1]. This prototype project is intended to inspire students to recycle and reuse items around their homes and create something beneficial. Upon completion of the background research phase students transition to the prototype design, where students analyze the crucial functions of arm and develop a working cardboard prototype which will later be 3D printed and assembled. Next step would be to analyze the muscles, which normally control hand movements and connect them to EMG (Electromyography) sensors [2]. Signals from these sensors will be read by Arduino which in turn will activate the assigned motors in the prosthetic hand.

Engineering design process will be applied as extracurricular activity at first, however will later be developed and implemented as instructional module for the 9th and 10th grades. Students will be evaluated by the following outcomes: understanding of how to create an actuated hand prototype from cardboard, use of newspaper and popsicle sticks to finish working prototypes, 3D printing and the groundwork for excellent printing of prosthetics arm parts, assembling principles, and measuring muscle activation via electric potential (EMG), and converting signals from muscles into actual movements in the prosthetic hand. The final goal will be a successful execution of a given daily task such as picking up a piece of paper or paper bills or coins, holding a water bottle or a pen/pencil/paint brush, or gripping a toothbrush. Students will be expected to write a report with detailed description of all steps taken during the development of the prosthetic limb. 
Once we perfect the prosthetic arm, the same process can be applied to development of other limbs such as arms or legs. This project will benefit patients with disabilities and improve their day-to-day lives at a fraction of the cost of current solutions.

\section{Motivation}

The purpose of this project is to help low-income families' children who have a disabled hand and to expand students' awareness of societal needs. With this project, students demonstrate the learning and knowledge gained from the different sources, such as using Arduino board to design a myoelectric prosthetic arm. It is therefore important to show the students that they have studied well and applied their knowledge to solve the problems in the real world, which is meaningful to them. During the project, there are time and budget limits. The project's resource questions are how to create a prosthetic arm, how to use EMC sensors with the prosthetic arm. To create the prosthetic arm, students use the 3D printing and lay a groundwork for excellent printing of the prosthetics arm parts. Using the EMG sensor, students measure muscle activation via electric potential referred to as electromyography (EMG) and use Arduino to control the 3D prosthetic arm.

\section{Methodology}

\section{Overview}

The design process contains a series of steps that engineers follow when they are trying to solve real world problems, methodical approach to problem solving. The basic function of an engineering design is to create an item that solves a problem.

Students choose at least one task for their designed hand to complete.

- Pick up a piece of paper or paper bill

- Pick up a coin

- Hold a water bottle

- Grip a toothbrush

- Hold a pen/pencil/paint brush

\section{Preparation for 3D printing}

Students first lay the groundwork for excellent printing of the prosthetics arm parts [3]. To change the basic design, students need to use a toolbox called Tinkercad. Tinkercad is introduced in class. 


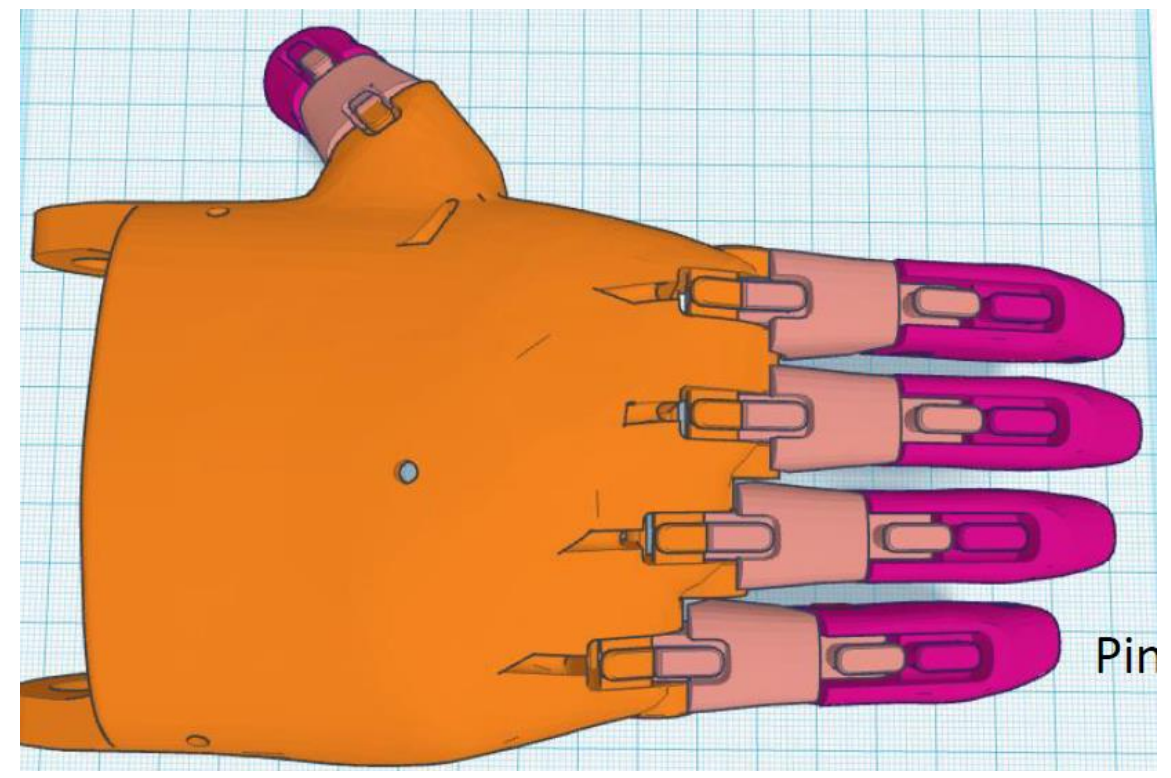

Figure 1: e-NABLE Phoenix Hand v2

(http://www.thingiverse.com/thing:1453190) by EnableCommunityFoundation [4].

\section{Effective 3D printing of eNABLE hands}

Because 3D printers fabricate objects from thin layers of plastic, there is a grain to the structure of the printed parts, much like there is grain in wood. In addition, the "grain" of the print needs to be oriented to maximize the strength of the part for building the hand and fingers, so many of the parts used in the eNABLE hands need to be printed in a particular orientation [4]. For example, designing a mechanical arm when student bends his/her wrist down, the fingers come down, too, because they are being pulled. After that, when the student returns his/her wrist to a straight position, the fingers open up and go back to normal.

\section{Using Arduino to control 3D Prosthetic arm based on Myoelectric signal}

The Myoware Sensor from Advancer Technology is an open source EMG sensor that measures, filters, rectifies, and amplifies muscle electrical activity. An Arduino Uno clone from Sparkfun is used to read data from the Myoware Sensor. The Myoware board acts by measuring the filtered and rectified electrical activity of a muscle, and outputs 0-5 Volts depending on the amount of activity in the selected muscle. Arduino Uno has a built-in 10 bit ADC (Analog to digital converter) that reads the analog signal generated by the Myoware Sensor. The 10-bit number is then scaled between $0-5 \mathrm{~V}$, which is used to control the servo. The voltage threshold for sensor actuation is $2.5 \mathrm{~V}$. When the threshold voltage is exceeded, an HXT500 servo motor will rotate 180 degrees; otherwise it will remain at 0 Degrees. The servo will be attached to a Phoenix Hand $\mathrm{V} 2$ and be able to actuate one of its fingers, allowing the finger to close and open. The final project design (Figure 1) and circuitry (Figure 2) is shown below. 


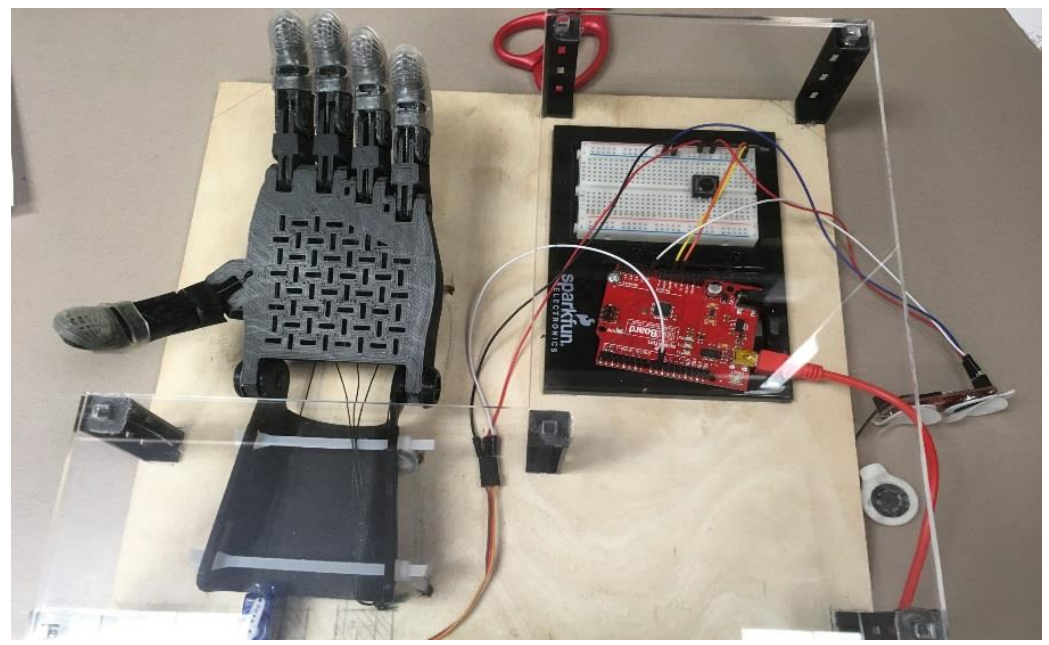

Figure 1. Phoenix hand V2, Servo Motor, Arduino Board, Bread Board, and EMG Sensor

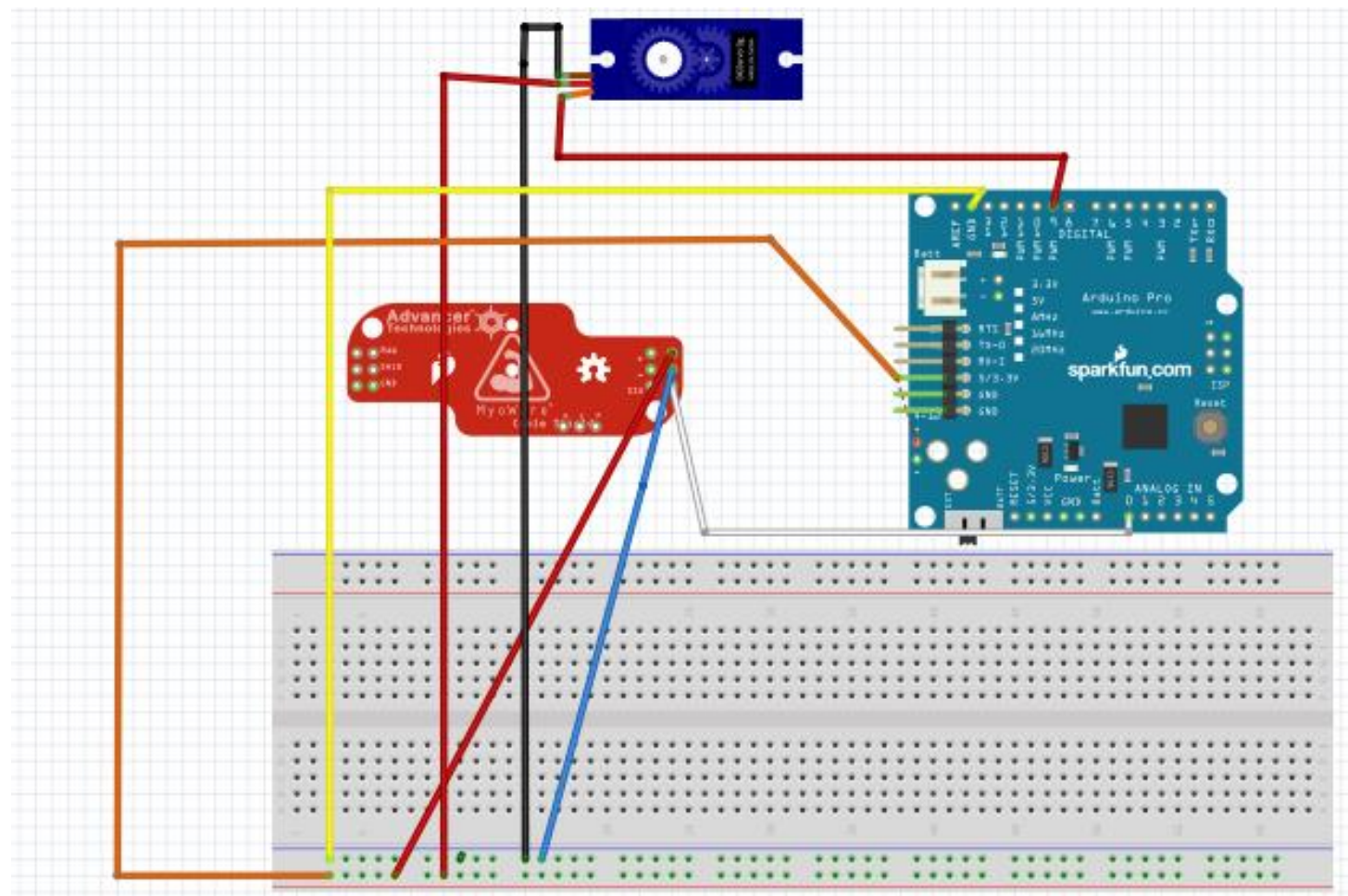

Figure 2. Circuit to connect the EMG sensor and the servo motor to the Arduino

\section{Materials List}

The 3D hand project requires a circuit board, several cables, an Arduino Mega/Uno kit, EMG Sensor, 3D hand assembly kit (Table 1). 
Table 1: Components and prices

\begin{tabular}{|l|r|}
\hline Component & Approximate Cost \\
\hline MyoWare Muscle Sensor Development Kit & $\$ 79.95$ \\
\hline Arduino Nano V3.0, Elegoo Nano board & $\$ 11.86$ \\
\hline HXT500 Micro Servo & $\$ 3.66$ \\
\hline $\begin{array}{l}\text { eBoot 120 Pieces Breadboard Jumper Wires Ribbon Cables Kit Wire 40 } \\
\text { Pin }\end{array}$ & $\$ 7.69$ \\
\hline Phoenix Hand by eNABLE Assembly Materials Kit & $\$ 30.00$ \\
\hline Total & $\$ 133.16$ \\
\hline
\end{tabular}

\section{Procedure}

Step 1. Install the Arduino Software IDE on Windows from this website: https://www.arduino.cc/en/Guide/Windows

Step 2. Wire the Arduino and servo with a breadboard and EMG Sensor

1. Connect the brown wire of the servo to GND on the Arduino

2. Connect the red wire of the servo to $5 \mathrm{~V}$

3. Connect the yellow wire of the servo to Digital 9

4. Connect a wire to any of the pins of a button to $5 \mathrm{~V}$

5. Connect a wire on the same side of the button as part $d$ (with the connections) to Digital 7

The Arduino code below is used to turn the servo 180 degrees when the button is pushed. This is to test the servo and to show that it will be capable of pulling a string.

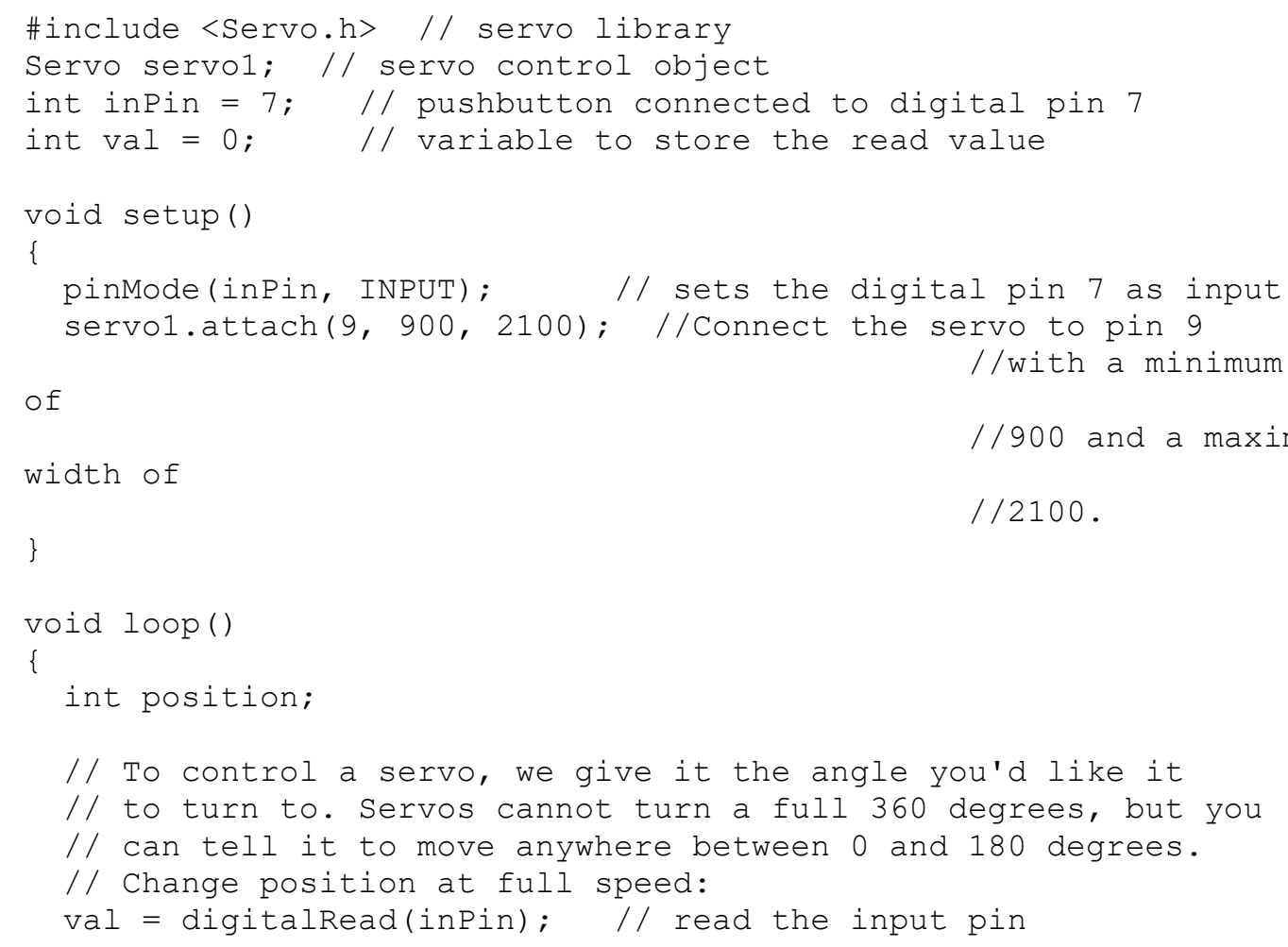




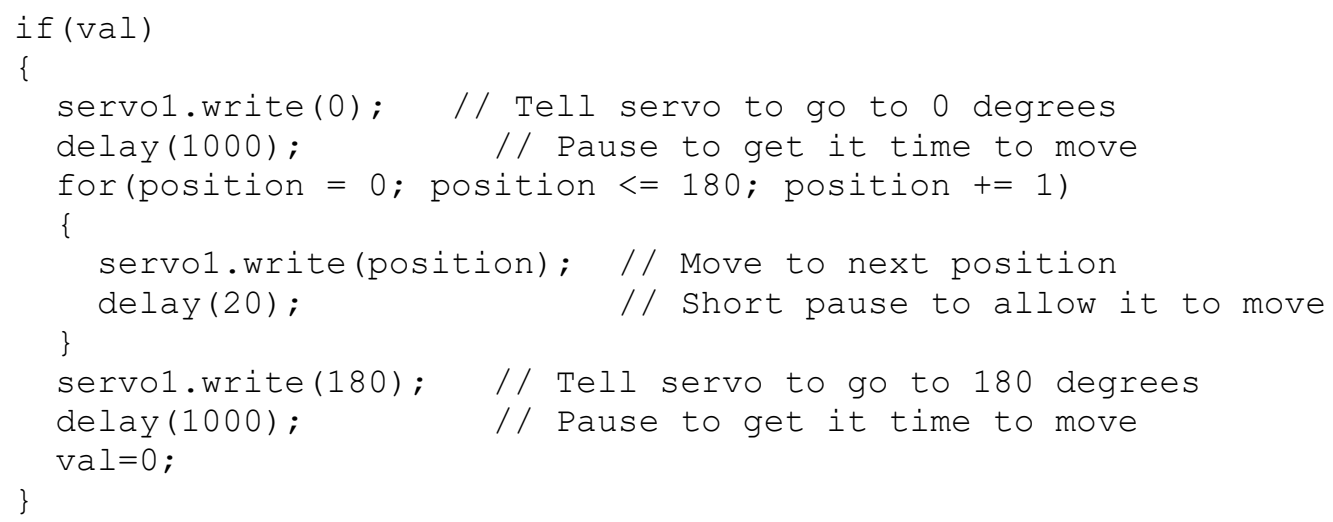

6. Wire the EMG

- Attach the + pin on the EMG to 5V

- Attach the - pin on the EMG to GND

- Attach the SIG pin on the EMG to A0

Step 3. Use Arduino with EMG sensor Schematic

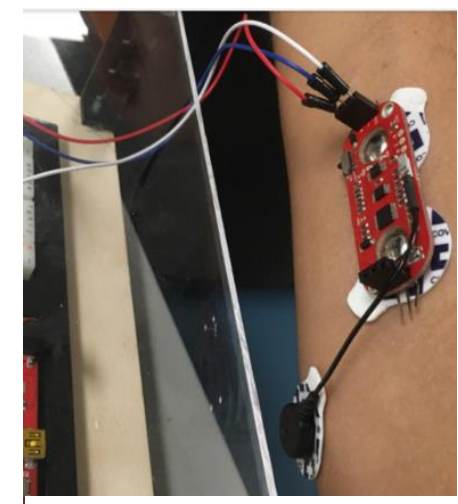

Figure 3: EMG Sensor

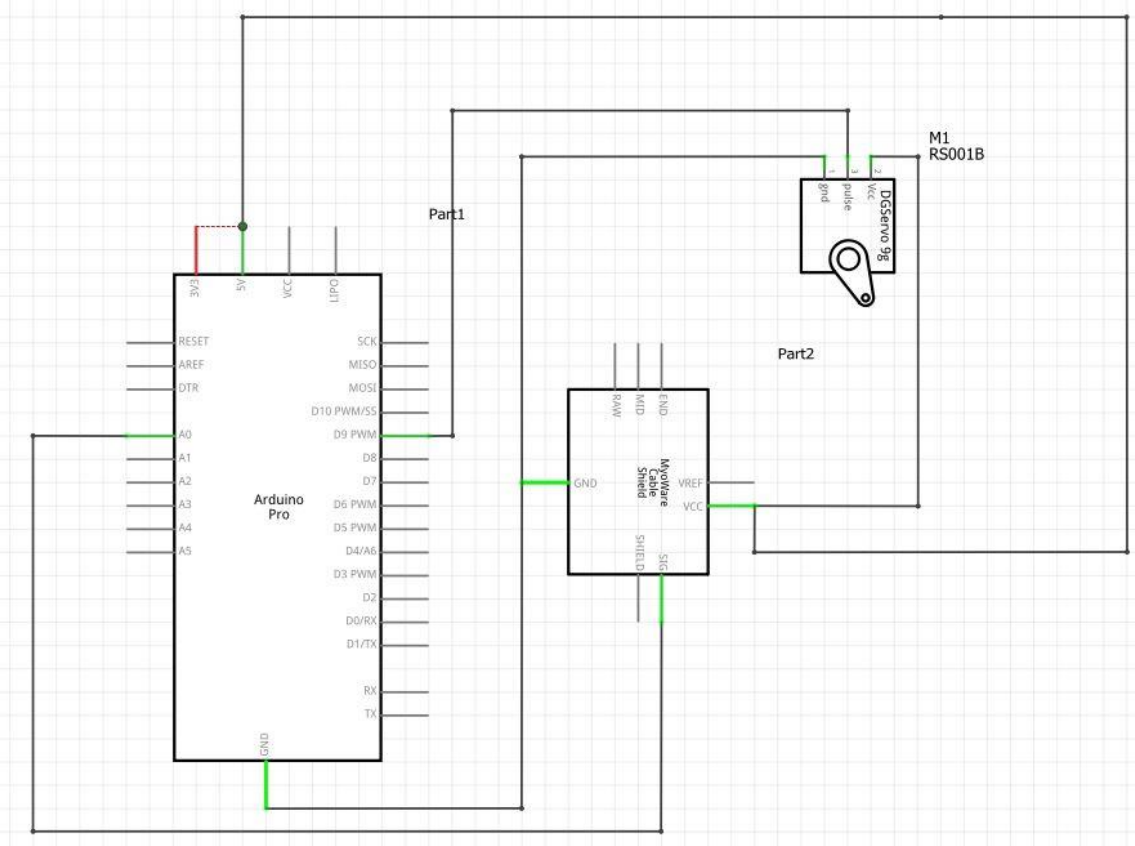

Figure 4: Arduino Schematic

Attach EMG electrodes to the board and attach them to an arm similar to the picture below. Make sure to put it on the green area, as this is the center of the muscle and will have the best results. Connect the last electrode to a different muscle or bone. 
Below is Arduino code used for testing the EMG sensor.

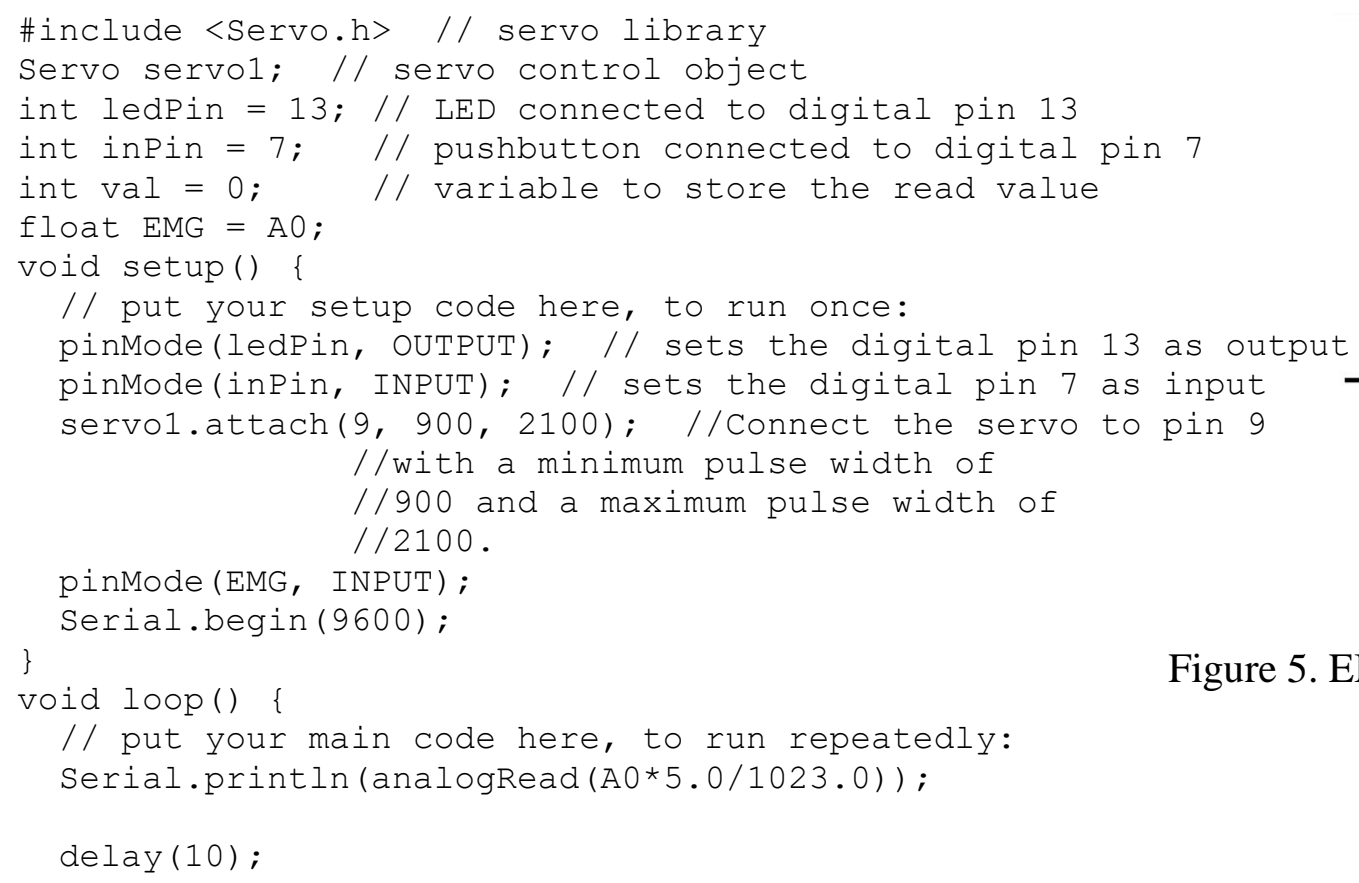

One of the EMG sensors is used to turn the motor 180 degrees. When a person's arm flexes, the string is pulled by the servo to close the fingers.

\section{Outreach Activities}

The project will be used at a STEM festival, which is organized to exhibit our science, technology, engineering and mathematics projects to general public. The STEM festival enables students to demonstrate their projects in front of peers, parents and other guests, thus inspiring and attracting other students to STEM fields. The use of different resources allows students to learn and work independently and as a part of a team, and allows the teacher to become more of a facilitator in the classroom.

\section{Results and Conclusions}

The main objective is this project is to design a prosthetic hand using $3 \mathrm{D}$ printed parts, and control them using Arduino based on myoelectric inputs from the patients arm. The first part of the project would be to control one of five fingers by flexing the muscle attached to the EMG electrodes. As we proceed through the development we will add more functionality to the hand.

This project will engage students in solving real life problems that will benefit people with disabilities.

\section{Acknowledgements}

This work was supported by a grant from the National Science Foundation's Research Experience for Teachers (RET) Program (Award No. 1300779). Any opinions, findings, and 
conclusions or recommendations expressed in this material are those of the authors and do not necessarily reflect the views of the National Science Foundation.

We would like to thank Mr. Zhong Thai for assistance with programming and Dr. Pilwon Hur for access to equipment and information needed to complete the project.

\section{References}

[1] Using Arduino to Design a Myoelectric Prosthetic, K.Talbot, [Online]. Available: http://digitalcommons.csbsju.edu/honors theses/55/ [Accessed July 13, 2017].

[2] MyoWare Muscle Sensor Kit - learn.sparkfun.com. [Online]. https://learn.sparkfun.com/tutorials/myoware-muscle-sensor-kit/all?print=1 [Accessed July 13, 2017].

[3] Medical Stockings, [Online] Available: http://www.medicalstockingsonline.com/Medivensleeves-fitting-chart-menu.php. [Accessed:July 12, 2017].

[4] Tips for Successful Prints - Enabling The Future, [Online] Available: http://enablingthefuture.org/resources-2/tips-for-successful-prints/ [Accessed July 15, 2017]. 